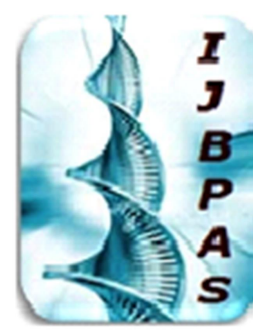

International Journal of Biology, Pharmacy and Allied Seiences (IJBPAS) 'A Bridge Betuen caboratory and QRader'

Www.ijbpas.com

\title{
THE CURRENT STATUS OF ANGIOTENSIN II IN PATIENTS WITH SHOCK: A REVIEW OF THE LITERATURE \\ NAWAF MOHAMED ALOTAIBI
}

Assistant Professor, Department of Pharmaceutics, Faculty of Pharmacy, Northern Border University, Rafha, Saudi Arabia

*Corresponding Author: Dr. Nawaf Mohamed Alotaibi: E Mail: nawa-2@hotmail.com; Nawaf.AL-Otaibi@nbu.edu.sa

Received 12 ${ }^{\text {th }}$ March 2021; Revised 15 ${ }^{\text {th }}$ April 2021; Accepted $31^{\text {st }}$ May 2021; Available online $1^{\text {st }}$ March 2022 https://doi.org/10.31032/IJBPAS/2022/11.3.5941

\begin{abstract}
Angiotensin II is an octapeptide with potent vasoconstrictor activities. The recent publication of favorable data from ATHOS-3 trial in 2017 was followed by FDA priority review approval of GIAPREZA (LJPC-501) for management of hypotension in states of vasodilatory shock. Aiming to summarize the current status of clinical research on the therapeutic use of Angiotensin II in patients with shock, we searched for published and ongoing clinical trials, and reviewed the cornerstone research articles on the topic. It appears that the existing research on clinical use of Angiotensin II is rather scarce, however, with the recent approval under priority review, a boost in clinical research is highly anticipated, in order to provide further insight into the potential implications of the synthetic octapeptide in various refractory hypotensive states.
\end{abstract}

Keywords: Refractory circulatory failure, sepsis, vasopressors, peptide hormone, FDA, novel therapeutics

\section{INTRODUCTION}

Shock is a devastating syndrome of acute or hyperacute onset with considerable morbidity and mortality [1], and is characterized by signs and symptoms that result from a systemic response to multi- organ hypoperfusion with inherent compromise in end-organ metabolic requirements at cellular level, and hallmarked by hypotension [2]. In the current clinical setting, as reflected in a 
number of recent guideline recommendations [3], whenever vasopressor agents are indicated, norepinephrine (NE) remains the drug of choice for management of hypotension in patients with distributive (i.e. vasodilatory) [4], as well as cardiogenic [5], or other forms of shock. Despite the vast amount of clinical experience with, and commonplace application of NE, its inadvertently unfavorable safety profile [6] delimits utility in the care of a patients suffering from shock [7], which has drawn the interest of clinicians and investigators toward the search for a novel vasopressor agent. Arginine vasopressin was initially considered an option, until its cardiac toxicity was recognized. The only viable alternative appeared to be Angiotensin II [8]. Commonly conceptualized as a key member of the rennin - angiotensin aldosterone system (RAAS), Angiotensin II is a naturally occurring octapeptide hormone with potent vasoconstrictor activities [9]. In December 2017, FDA priority review approved the use of synthetic human angiotensin II (marketed as GIAPREZA, proprietary formulation: LJPC-501) for increasing blood pressure in states of septic and other forms of distributive shock. The purpose of this review is to briefly summarize the current and ongoing clinical research on
Angiotensin II as a novel agent for use in patients with shock.

\section{MATERIALS AND METHODS}

We searched the electronic databases MEDLINE and EMBASE via Ovid for English titles and abstracts using the terms (angiotensin OR angiotensin II OR Ang2) AND (shock OR circulatory failure OR sepsis OR septic) to identify published clinical controlled trials on the use of angiotensin II in patients with septic or other forms of shock. The full paper forms of included abstracts were retrieved and reviewed. References provided in selected review articles were further hand-searched for additional relevant citations. We also searched ClinicalTrials.gov for recent or ongoing (yet unpublished) trials using "Angiotensin II" and "shock" as entry items for the "Intervention/treatment" and "Condition or disease" fields, respectively. The acquired data were analyzed.

\section{RESULTS}

The contemporary literature on therapeutic use of Angiotensin II appears to be scarce. According to our search results, to date, there are only two published randomized controlled trials, namely, the original ATHOS pilot [10] and ATHOS-3 [11] trials, and three ongoing clinical trials (Clinicaltrials.gov, NCT 03302650; NCT 03245528; and NCT 03623529; all currently recruiting participants) evaluating the efficacy of ATII in states of shock. 
Additionally, we identified 2 prematurely interrupted trials failing to recruit the estimated number of participants and therefore not reporting results (Clinicaltrials.gov, NCT 00711789; and NCT 03431077).

\section{DISCUSSION}

\section{Historical insights}

Since its synthesis [12] and purification [13], angiotensin II (ATII) has been experimented with in both animal and human subjects. It has been widely recognized that ATII is far more potent and safer [14] than other available vasopressor agents, which were notorious for narrow therapeutic indices (particularly norepinephrine). Despite a long history of experimental and clinical experience with ATII dating back to 1960's, high quality body of evidence has been lacking until recently, largely due to inhomogeneous study design, with case series and nonrandomized trials dominating the published literature [15]. Furthermore, the clinical implications of the vasoactive octapeptide received wider recognition in European countries than in US. Most notably, del Greco et al. [16] reported on their clinical experience with 21 patients admitted with shock from various etiologies (10 patients with uremia and dialysis resulting in severe fluid shifts, 6 with bacteremia, 2 with acute coronary syndrome, 2 with barbiturate poisoning, and 1 with intracranial hemorrhage) in 27 separate encounters [16]. The authors administered ATII after discontinuing other vasopressors as well as in combination with norepinephrine (in 5 patients) and were able to elicit excellent recovery of blood pressure in the majority of patients (15 cases), thereby concluding, that compared to norepinephrine, ATII performed more efficiently at raising blood pressure and was the most effective pressor agent available at that time. Particularly promising results were documented for patients with septic shock. Similar outcomes were observed by Derrick et al. [17], who summarized data from 10 patients with shock from variable etiologies, who were treated with ATII. The authors concluded that ATII was safe and effective for increasing blood pressure and reducing heart rate, with comparable and somewhat superior effects compared to norepinephrine [17].

\section{The renewed interest}

The recent interest in reviving the use of ATII began with ATHOS pilot study [10]. In search for a novel vasopressor agent that would lack the prone-to-tachyphylaxis properties of catecholamines and cardiotoxic adverse effects of vasopressin, the authors arrived at the idea of revisiting the implications of the long-forgotten octapeptide, resulting in the first prospective randomized controlled trial to evaluate the treatment with ATII. Twenty 
patients with distributive shock and under standard of care management, were randomized to receive either add-on ATII infusion or placebo, with 10 patients included in each group. The ATII arm demonstrated marked reduction in norepinephrine (NE) dose requirements. Despite the non-significant difference in 30 -day mortality between groups (50\% vs. $60 \%$ ), the drug was well tolerated, and the initial intent of the pilot study was to establish a working drug dose to commence with and facilitate further clinical research [10]. Following these intriguing findings, the authors proceeded with an international multicenter successor trial held in 9 countries across North America, Europe and Australia. ATHOS-3 was a doubleblind, randomized controlled phase 3 clinical trial that included a total of 344 patients [11]. Twenty-three patients dropped out of the study due to rapid improvement or decline of condition, physician decision, or consent withdrawal. The 321 patients who proceeded with the trial were randomized to either add-on ATII (163) or placebo (158). The ATII treatment arm demonstrated statistically significant increase in mean arterial pressure compared to placebo $(12.5 \mathrm{~mm} \mathrm{Hg}$ vs. $2.9 \mathrm{~mm} \mathrm{Hg}$ ) in the initial 3 hours of administration, thus meeting the primary end-point. Seventy percent of patients in the ATII infusion group achieved a mean artery pressure (MAP) of $\geq 75 \mathrm{~mm} \mathrm{Hg}$ or increased MAP by $\geq 10 \mathrm{~mm} \mathrm{Hg}$, compared to only $23 \%$ in the placebo group. The ATII arm also performed well regarding background vasopressor support reduction in the first 48 hours of administration, thus meeting the additional end-point. Despite the favorably lower Sequential Organ Failure Assessment (SOFA) scores [18] at 48 hours compared to placebo, the change in score was not significantly different between groups. Furthermore, higher absolute heart rates were observed in the ATII infusion arm. Given that that tachycardia is well-known poor prognosticator that increases mortality in critically ill patients [19], the latter observation most desirably should be avoided. Additionally, despite the initial intent of presenting results in a population with various forms of vasodilatory shock states, the majority of patients $(\sim 80 \%)$ turned out to be suffering from sepsis and the likelihood of sepsis could not be excluded in an additional $10 \%$ of patients. Thus, it would not be wise to generalize the findings to other vasodilatory shock states [20]. The findings of ATHOS-3 met FDA priority review approval of ATII as proof of concept, given that clinical efficacy and safety were granted by the aforementioned trials [10]. A post-hoc study analyzing the results of ATHOS-3 evaluated the impact of ATII on acute kidney injury (AKI) that 
required administration of renal replacement. The 28-day survival and mean arterial pressure response were higher in ATII group and more patients discontinued renal replacement [21]. These findings prompt investigation of other potential benefits related to blood pressure recovery achieved by ATII, as such effects remain largely unknown.

A systematic review of all published data on intravenously administered ATII in human subjects included the RCTs ATHOS and ATHOS-3, case-control studies and case reports [15]. In addition to effectiveness of ATII, the authors also acknowledged the potential role of ATII in the treatment of ACE inhibitor overdose, and management of patients with cardiogenic shock, who were previously exposed to ACE inhibitors. These populations were not addressed in the randomized trials above.

\section{Ongoing Clinical Research}

The Angiotensin in Septic Kidney Injury Trial (ASK-IT) was initiated in 2009 as a crossover RCT intending to investigate the effects of Angiotensin II on renal failure (Clinicaltrials.gov, NCT 00711789). However, the trial never achieved the estimated recruitment goal of 12 patients and the outcomes were not reported.

The ANGSTROM trial (Clinicaltrials.gov, NCT 03302650) is a 2017 phase III pilot study initiated in Cairo, Egypt, aiming to investigate the effects of ATII on macroand micro- circulation in patients with shock. Utilizing side stream dark field imaging optical probe (Microscan), the investigators plan to image tongue vasculature after intravenous administration of ATII and measure mean flow index as a primary outcome and proportion and density of perfused vessels as well as a number of other clinically relevant variables as secondary outcomes. The study has not initiated patient recruitment yet.

Another trial financed by La Jolla Pharmaceutical Company, and initially designed to be a phase II open-label, multicenter study initiated in North Carolina, US in 2018 (Clinicaltrials.gov, NCT 03431077), was set to investigate the effects of ATII infusion in pediatric patients with refractory, catecholamineresistant hypotension $(\mathrm{CRH})$ not responding to adequate fluid and vasopressor support, with two enlisted primary outcomes as the mean arterial pressure (MAP) and sum NE equivalent dose at 2 hours after infusion. With an estimated sample size of 30 participants, however, the trial was prematurely ended in April 2018, failing to recruit adequate number of patients (only two children participated). Subsequently, in August 2018, La Jolla Pharmaceutical Company initiated a randomized, placebo-controlled, double-blinded, multicenter pediatric 
efficacy and safety trial in Belgium and Lithuania, which is currently recruiting, aiming at 100 participants, and estimated completion in 2023 (Clinicaltrials.gov, NCT 03623529). The primary outcome is the proportion of patients with more than $50 \%$ reduction in $\mathrm{NE}$ equivalent doses within three hours of administration. Further attempts are made to expand the access of shock patients to Angiotensin II (NCT 03245528)

All of the aforementioned ongoing trials use ATII as an add-on therapy and compare to saline placebo. While ANGSTROM trial is mainly pathophysiology oriented, the latter two trials were attempts to recapture the ATHOS findings in pediatric patients to broaden the ATII research population. It is highly anticipated, that in the near future the clinical trials will be aiming at evaluation of the add-on ATII treatment on the background of standard of care vasopressors (similar to the ATHOS-3 trial), and examining the various effects on different organ systems, and most desirably, shock states of other vasodilatory (i.e. other than septic) as well as nonvasodilatory origin (e.g. cardiogenic, hemorrhagic etc.). As dosage, administration, and safety issues appear to approach settlement by entering the realm of evidence-based medicine, direct comparisons of ATII with norepinephrine will clearly settle the score with particular insights into specific patient populations who might additionally benefit from one or the other vasopressor agent [8].

\section{Safety Concerns}

In ATHOS-3 trial the overall occurrence of adverse events was reported to be as common with ATII as with placebo, and the causes of discontinuation were similar [12]. Most concerning findings were those related to thrombotic events (largely deep venous thromboses) which were more common in ATII group (13\%) than in placebo group (5\%). Other notable adverse events were related to thrombocytopenia, tachycardia, fungal infections, delirium, acidosis, hyperglycemia and peripheral ischemia.

A systematic review evaluating the safety of ATII with intravenous route included 1,124 articles published from 1941 to 2016 , altogether encompassing 31,281 subjects, who were administered the drug for various purposes including physiology and efficacy studies in healthy volunteers, healthy pregnancies, various medical conditions as well as shock of different etiologies [16]. Two deaths occurred (excluding the patients with oncologic morbidity and refractory shock) and fewer than 300 adverse events were reported. The documented deaths, both reported in 1965, were not linked to shock, and encompassed one case of intracranial hemorrhage in a healthy volunteer who was receiving a 6- 
day course of continuous IV ATII infusion, and a severe left ventricular failure manifested as acute decompensation of preexisting heart failure. Despite these 2 dramatic cases, no other serious adverse events or morbidities could be identified in linked to ATII administration. However, a potential risk of exacerbating preexisting asthma and CHF was recognized. Despite the much shorter durations, lower doses and interrupted administration regimens commonly reported in the literature, the authors concluded that an acceptable safety profile could be achieved.

\section{CONCLUSION}

Angiotensin II infusion appears to be a viable option for patients with septic shock and potentially useful in other vasodilatory as well as non-vasodilatory hypotensive conditions. The current published evidencebased research has largely been limited by the two randomized controlled trials evaluating the add-on benefits of ATII. Nonetheless, the FDA approval of GIAPREZA will likely boost the research. To that end, currently there are 2 ongoing clinical trials comparing ATII with placebo, one of which aims to reconstruct the findings of ATHOS in pediatric patients with shock. Further clinical trials aiming for direct comparisons of ATII and norepinephrine (NE) are most likely to settle the score. Some patient populations may gain additional benefits from ATII or
$\mathrm{NE}$ and this remains to be investigated. The data from post-hoc analysis of ATHOS-3 trial are intriguing and mandate additional insights into the trial results as well as evaluation of benefits for other organ systems (e.g. renal failure, acute decompensated heart failure) in new trials. ATII administration appears to be largely safe with an acceptable rate of adverse events.

\section{REFERENCES}

[1] Knoop, S.T., Skrede, S., Langeland, N., \& Flaatten, H.K. (2017). Epidemiology and impact on allcause mortality of sepsis in Norwegian hospitals: A national retrospective study. PLoS ONE, 12(11), e0187990. DOI: 10.1371/journal.pone.0187990

[2] Bonanno, F.G. (2011). Clinical pathology of the shock syndromes. Journal of Emergencies, Trauma and Shock, 4(2), 233-243. DOI: 10.4103/0974-2700.82211

[3] Rhodes, A., Evans, L.E., Alhazzani, W., Levy, M.M., Antonelli, M., Ferrer, R., Kumar, A., Sevransky, J.E., Sprung, C.L., Nunnally, M.E., Rochwerg, B., Rubenfeld, G.D., Angus, D.C., Annane, D., Beale, R.J., Bellinghan, G.J., Bernard, G.R., Chiche, J.D., Coopersmith, C., De Backer, D.P., French, C.J., Fujishima, S., Gerlach, H., Hidalgo, 
J.L., Hollenberg, S.M., Jones, A.E.,

Karnad, D.R., Kleinpell, R.M., Koh,

Y., Lisboa, T.C., Machado, F.R.,

Marini, J.J., Marshall, J.C.,

Mazuski, J.E., McIntyre, L.A.,

McLean, A.S., Mehta, S., Moreno,

R.P., Myburgh, J., Navalesi, P.,

Nishida, O., Osborn, T.M., Perner,

A., Plunkett, C.M., Ranieri, M.,

Schorr, C.A., Seckel, M.A.,

Seymour, C.W., Shieh, L., Shukri,

K.A., Simpson, S.Q., Singer, M.,

Thompson, B.T., Townsend, S.R.,

Van der Poll, T., Vincent, J.L.,

Wiersinga, W.J., Zimmerman, J.L.,

\& Dellinger, R.P. (2017). Surviving

Sepsis Campaign: International

Guidelines for Management of

Sepsis and Septic Shock: 2016.

Intensive Care Medicine, 43(3), 304-377.

\section{https://doi.org/10.1007/s00134-}

017-4683-6

[4] van Diepen, S., Katz, J.N., Albert,

N.M., Henry, T.D., Jacobs, A.K.,

Kapur, N.K., Kilic, A., Menon, V.,

Ohman, E.M., Sweitzer, N.K.,

Thiele, H., Washam, J.B., \& Cohen,

M.G. (2017). Contemporary

Management of Cardiogenic Shock:

A Scientific Statement From the

American Heart Association.

Circulation 136(16), 232-268. DOI:

10.1161/CIR.0000000000000525
[5] Oda, S., Aibiki, M., Ikeda, T., Imaizumi, H., Endo, S., Ochiai, R., Kotani, J., Shime, N., Nishida, O., Noguchi, T., Matsuda, N., \& Hirasawa, H. (2014). Sepsis Registry Committee of JSICM: The Japanese guidelines for the management of sepsis. Journal of Intensive Care, 2(1), 55. DOI https://doi.org/10.1186/s40560-014$\underline{0055-2}$

[6] De Backer, D., Aldecoa, C., Njimi, H., \& Vincent, J.L. (2012). Dopamine versus norepinephrine in the treatment of septic shock: A meta-analysis. Critical Care Medicine, 40(3), 725. DOI: 10.1097/CCM.0b013e31823778ee

[7] Anantasit, N., Boyd, J.H., Walley, K.R., \& Russell, J.A. (2014). Serious adverse events associated with vasopressin and norepinephrine infusion in septic shock. Critical Care Medicine, 42(8), 1812-1820.

doi:10.1097/CCM.0000000000000333

[8] Dünser, M.W., \& Meier, J. (2017). Vasopressor hormones in shocknoradrenaline, vasopressin or angiotensin II: which one will make the race? Journal of Thoracic Disease, 9(7), 1843-1847. doi: $10.21037 /$ jtd.2017.06.127 
[9] Corrêa, T.D., Takala, J., \& Jakob, S.M. (2015). Angiotensin II in septic shock. Critical Care, 19(1), 98. DOI: 10.1186/s13054-0150802-3

[10] Chawla, L.S., Busse, L., BrashaMitchell, E., Davison, D., Honiq, J., Alotaibi, Z., \& Seneff, M.G. (2014). Intravenous angiotensin II for the treatment of high-output shock (ATHOS trial): a pilot study. Critical Care, 18(5), 534. doi: 10.1186/s13054-014-0534-9

[11] Khanna, A., English, S.W., Wang, X.S., Ham, K., Tumlin, J., Szerlip, H., Busse, L.W., Altaweel, L., Albertson, T.E., Mackey, C., McCurdy, M.T., Boldt, D.W., Chock, S., Young, P.J., Krell, K., Wunderink, R.G., Ostermann, M., Murugan, R., Gong, M.N., Panwar, R., Hästbacka, J., Favory, R., Venkatesh, B., Thompson, B.T., Bellomo, R., Jensen, J., Kroll, S., Chawla, L.S., Tidmarsh, G.F., \& Deane, A.M. (2017). Angiotensin II for the Treatment of Vasodilatory Shock. New England Journal of Medicine, 377(5), 419-430. DOI:

\subsection{6/NEJMc1714511}

[12] Rittel, W., Iselin, B., Kappeler, H., Riniker, B., \& Schwyzer, R. (1957). Synthese eines hochwirksamen Hypertensin

II-amids(L-Asparaginyl-L-arginylL-valyl-L-tyrosyl-L-isoleucyl-L-hi stidyl-L-prolyl-L-phenylalanin). Angewandte Chemie, 69(5), 179179.https://doi.org/10.1002/hlca.1 9570400317

[13] Bumpus, F.M., Schwarz, H., \& Page, I.H. (1957). Synthesis and Pharmacology of the Octapeptide Angiotonin. Science, 125(3253), 886-887.

DOI:10.1126/science.125.3253.886

[14] Gross, F., \& Turrian, H. (1960). Pharmacology of hypertensin and synthetic analogues. In Schachter M (ed): Polypeptides which affect smooth muscles and blood vessels. New York, US: Pergamon Press, Inc., p 137.

[15] Busse, L.W., Wang, X.S., Chalikonda, D.M., Finkel, K.W., Khanna, A.K., Szerlip, H.M., Yoo, D., Dana, S.L., \& Chawla, L.S. (2017). Clinical Experience with IV Angiotensin II Administration: A Systematic Review of Safety. Critical Care Medicine, 45(8), 1285-1294.

DOI: 10.1097/CCM.0000000000002441

[16] Del Greco, F. (1961). Clinical Experience with Angiotensin II in the Treatment of Shock. Journal of American Medical Association, 
178(10),

994.

doi:10.1001/jama.1961.030404900 20005

[17] Derrick, J.R., Anderson, J.R., \& Roland, B.J. (1962). Adjunctive Use of a Biologic Pressor Agent, Angiotensin, in Management of Shock. Circulation, 25(1), 263267. DOI: 10.1161/01.cir.25.1.263

[18] Vincent, J.L., Moreno, R., Takala, J., Willatts, S., De Mendonça, A., Bruining, H., Reinhart, C.K., Suter, P.M., \& Thijs, L.G. (1996). The SOFA (Sepsis-related Organ Failure Assessment) score to describe organ dysfunction/failure. On behalf of the Working Group on Sepsis-Related Problems of the European Society of Intensive Care Medicine. Intensive Care Medicine, 22(7), 707-710. DOI: 10.1007/BF017

[19] Parker, M.M., Shelhamer, J.H., Natanson, C., Alling, D.W., \& Parrillo, J.E. (1987). Serial cardiovascular variables in survivors and nonsurvivors of human septic shock: heart rate as an early predictor of prognosis. Critical care medicine, 15(10), 923-929.

DOI: $\quad 10.1097 / 00003246-$ 198710000-00006
[20] Hessler, M., Arnemann, P.H., Seidel, L., Kampmeier, T., Rehberg, S., \& Ertmer, C. (2017). ATHOS-3 and the knights of the round table - the search for the holy grail of vasopressors. Journal of Thoracic Disease, 9(9), 27882791.

doi:

$10.21037 /$ jtd.2017.08.29

[21] Tumlin, J.A., Murugan, R., Deane, A.M., Ostermann, M., Busse, L.W., Ham, K.R., Kashani, K., Szerlip, H.M., Prowle, J.R., Bihorac, A., Finkel, K.W., Zarbock, A., Forni, L.G., Lynch, S.J., Jensen, J., Kroll, S., Chawla, L.S., Tidmarsh, G.F., \& Bellomo, R. (2018). Outcomes in Patients with Vasodilatory Shock and Renal Replacement Therapy Treated with Intravenous Angiotensin II. Critical Care Medicine, 46(6), 949-957. DOI: 10.1097/CCM.0000000000003092 